\title{
Stability and Energy-Casimir Mapping for Integrable Deformations of the Kermack-McKendrick System
}

\author{
Cristian Lăzureanu (D) and Camelia Petrişor \\ Politehnica University of Timişoara, Department of Mathematics, Piața Victoriei No. 2, 300006 Timişoara, Romania \\ Correspondence should be addressed to Cristian Lăzureanu; cristian.lazureanu@upt.ro
}

Received 30 March 2018; Accepted 7 May 2018; Published 3 June 2018

Academic Editor: Alkesh Punjabi

Copyright (C) 2018 Cristian Lăzureanu and Camelia Petrişor. This is an open access article distributed under the Creative Commons Attribution License, which permits unrestricted use, distribution, and reproduction in any medium, provided the original work is properly cited.

\begin{abstract}
Integrable deformations of a Hamilton-Poisson system can be obtained altering its constants of motion. These deformations are integrable systems that can have various dynamical properties. In this paper, we give integrable deformations of the KermackMcKendrick model for epidemics, and we analyze a particular integrable deformation. More precisely, we point out two Poisson structures that lead to infinitely many Hamilton-Poisson realizations of the considered system. Furthermore, we study the stability of the equilibrium points, we give the image of the energy-Casimir mapping, and we point out some of its properties.
\end{abstract}

\section{Introduction}

Evolution equations represent models for describing phenomena that appear in physics, biology, chemistry, economy, and engineering. In many situations these evolution equations can be analyzed in the frame of the Lagrangian mechanics or the Hamiltonian mechanics. Furthermore, there are phenomena that are modeled by three-dimensional systems of differential equations, particularly HamiltonPoisson systems. Such systems can be perturbed in order to obtain a desired behavior. A way to perturb a threedimensional Hamilton-Poisson systems consists in alteration of its constants of motion. This method leads to integrable deformations of the initial system.

In recent papers, integrable deformations of some particular Hamilton-Poisson systems were analyzed. In [1], observing that the constants of motion of the Euler top determine its equations, integrable deformations of the Euler top were given. In [2], integrable deformations of the three-dimensional real valued Maxwell-Bloch equations were obtained by altering the constants of motion of the considered system. In the same manner, in [3], integrable deformations of the Rikitake system were constructed. These integrable deformations can be viewed as controlled systems and, in consequence, a study of modifications in their dynamics can be performed. Moreover, the integrable deformations of the above systems are also Hamilton-Poisson systems. Consequently, they can be analyzed from some standard and nonstandard Poisson geometry points of view [4].

The study of a three-dimensional Hamilton-Poisson system from some standard and nonstandard Poisson geometry points of view tries to answer the following open problem formulated by Tudoran et al. [4]: "Is there any connection between the dynamical properties of a given dynamical system and the geometry of the image of the energy-Casimir mapping, and if yes, how can one detect as many as possible dynamical elements (e.g., equilibria, periodic orbits, homoclinic and heteroclinic connections) and dynamical behavior (e.g., stability, bifurcation phenomena for equilibria, periodic orbits, homoclinic and heteroclinic connections) by just looking at the image of this mapping?" Affirmative answers were given for some particular systems [5-9]. In these cases the image of the energy-Casimir mapping $\mathscr{E} \mathscr{C}=(H, C)$, where $H$ is the Hamiltonian and $C$ is a Casimir function, is a closed subset of $\mathbb{R}^{2}$, namely, the convex hull of the images of the stable equilibrium points through $\mathscr{E} \mathscr{C}$. Furthermore, the images of the equilibrium points through the energy-Casimir mapping give an algebraic partition of the set $\operatorname{Im}(\mathscr{E} \mathscr{C})$, and the orbits of these systems are bounded. On the other hand, the image of the energy-Casimir mapping can be $\mathbb{R}^{2}([10,11])$, 
and other connections were observed (for example, there are unbounded orbits). In [12], taking into account these facts, some questions regarding the connections between the dynamics of a Hamilton-Poisson systems and the associated energy-Casimir mapping were asked. We recall some of them: "are the observed properties in every case when $\operatorname{Im}(\mathscr{E} \mathscr{C}) \subsetneq$ $\mathbb{R}^{2}$ true?" or "can $\operatorname{Im}(\mathscr{E} \mathscr{C})$ be a nonconvex set? If yes, do the observed properties remain true?" One of the goals of this paper is to give some answers to these questions.

The finding of some counterexamples assumes the study of many Hamilton-Poisson systems, and how we have already seen such systems can be obtained using integrable deformations of known integrable systems. Moreover, because the constants of motion $H$ and $C$ of the above-mentioned systems are polynomials, it is a good idea to analyze systems that have nonpolynomials constants of motion. Such a system is the well-known system introduced in 1927 by Kermack and McKendrick [13] and brought back in attention by Anderson and May, in 1979 [14]. The Kermack-McKendrick system and its generalizations were widely investigated. We mention a very short list of works [15-17]. We also notice the applications of such type of systems in health, networks, informatics, economics, and finance (see, for example, [18] and references therein).

The paper is organized as follows. In Section 2, we recall the Kermack-McKendrick model and we give integrable deformations of this system. In Section 3, we analyze a particular integrable deformation of the Kermack-McKendrick system. More precisely, we point out two Poisson structures and, in consequence, we obtain two Hamilton-Poisson realizations of the considered system. In addition, using these structures, we construct infinitely many Hamilton-Poisson realizations of our system. Furthermore, we study the stability of the equilibrium points, we give the image of the energyCasimir mapping, and we point out some of its properties. The conclusions are presented in the last section.

\section{Integrable Deformations of the Kermack-McKendrick System}

Following [1], in this section, we give integrable deformations of the Kermack-McKendrick system. First, we recall the epidemic model introduced by Kermack and McKendrick [13] (for details, see also [19]).

In the mathematical theory of epidemics, a basic model is given by the Kermack-McKendrick system. This model intends to describe the spread of the infection within the population as a function of time. It is considered that the total population is constant, and it is divided into three distinct groups. First group is formed by individuals who can catch the disease, named the susceptibles. At a moment $t$ their number is $S(t)$. The second group, the infected population, consists in individuals who have the disease and can transmit it. Their number is $I(t)$. Finally, the group of the removed subjects, in number of $R(t)$, formed by those who had the disease, cannot be reinfected and cannot infect other individuals. In order to obtain the evolution equations, some assumptions were made. Firstly, the gain in the infective group is at a rate proportional to the number of infected subjects and susceptibles, that is, $a S(t) I(t)$, where $a>0$ is the infection rate. The susceptibles are lost at the same rate. Furthermore, the rate of removal of the infected subjects to the removed group is proportional to the number of the infected subjects, that is, $b I(t)$, where $b>0$ is the removal rate of the infected subjects. In addition, the incubation period is negligible, and every pair of individuals has equal probability of coming into contact with one another. Therefore, the following equations were deduced:

$$
\begin{aligned}
& \frac{d S}{d t}=-a S I \\
& \frac{d I}{d t}=a S I-b I \\
& \frac{d R}{d t}=b I .
\end{aligned}
$$

We denote $S:=x, I:=y, R:=z$. Then the KermackMcKendrick system is written as follows:

$$
\begin{aligned}
& \dot{x}=-a x y \\
& \dot{y}=a x y-b y \\
& \dot{z}=b y,
\end{aligned}
$$

where $a, b$ are positive constants.

It is obvious that a constant of motion is given by the total number of individuals; namely,

$$
I_{1}(x, y, z)=x+y+z .
$$

We recall the second constant of motion

$$
I_{2}(x, y, z)=\ln x+\frac{a}{b} z .
$$

Differentiating the above constants of motion we obtain

$$
\begin{aligned}
\dot{x}+\dot{y} & =-\dot{z}, \\
\frac{\dot{x}}{x} & =-\frac{a}{b} \dot{z},
\end{aligned}
$$

and considering $\dot{z}=b y$, we get system (2). Therefore the constants of motion (3), (4) generate system (2). This property allows us to obtain integrable deformations of system (2) by alteration of its constants of motion [1].

Consider as constants of motion the functions

$$
\begin{aligned}
& \widetilde{I}_{1}(x, y, z)=x+y+z+\alpha(x, y, z), \\
& \widetilde{I}_{2}(x, y, z)=\ln x+\frac{a}{b} z+\beta(x, y, z),
\end{aligned}
$$


where $\alpha, \beta$ are arbitrary differentiable functions. As above, we obtain that these functions generate the following system:

$$
\begin{aligned}
\dot{x}= & -a x y+b x y\left(\partial_{y} \beta-\partial_{z} \beta\right)-a x y \partial_{y} \alpha \\
& -b x y\left(\partial_{y} \alpha \partial_{z} \beta-\partial_{z} \alpha \partial_{y} \beta\right) \\
\dot{y}= & a x y-b y+a x y \partial_{x} \alpha-b y \partial_{z} \alpha+b x y\left(\partial_{z} \beta-\partial_{x} \beta\right) \\
& +b x y\left(\partial_{x} \alpha \partial_{z} \beta-\partial_{z} \alpha \partial_{x} \beta\right) \\
\dot{z}= & b y+b y \partial_{y} \alpha-b x y\left(\partial_{y} \beta-\partial_{x} \beta\right) \\
& -b x y\left(\partial_{x} \alpha \partial_{y} \beta-\partial_{y} \alpha \partial_{x} \beta\right),
\end{aligned}
$$

where $\partial_{x} f:=\partial f / \partial x$. If $\alpha$ and $\beta$ are constant functions, then (7) reduces to (2). Therefore, for any differential functions $\alpha$ and $\beta$, system (7) is an integrable deformation of the Kermack-McKendrick system.

Remark 1. In order to maintain constant the total population, the function $\alpha$ vanishes. In this case system (7) becomes

$$
\begin{aligned}
& \dot{x}=-a x y+b x y\left(\partial_{y} \beta-\partial_{z} \beta\right) \\
& \dot{y}=a x y-b y+b x y\left(\partial_{z} \beta-\partial_{x} \beta\right) \\
& \dot{z}=b y-b x y\left(\partial_{y} \beta-\partial_{x} \beta\right) .
\end{aligned}
$$

\section{Dynamical Properties of an Integrable Deformation of the Kermack-McKendrick System}

In this section, we consider some particular deformation functions, and we give some dynamical properties of the corresponding integrable deformation of the KermackMcKendrick system. First, we give Hamilton-Poisson realizations of this system that provides the geometric framework of our study. Furthermore, we study the stability of the equilibrium points. We also give some properties of the energyCasimir mapping associated with the considered system.

We consider the following deformation functions:

$$
\begin{aligned}
& \alpha(x, y, z)=\frac{g}{2} y^{2}+\frac{g}{2} z^{2}, \\
& \beta(x, y, z)=0,
\end{aligned}
$$

where $g \in \mathbb{R}$ is the deformation parameter. Then system (7) becomes

$$
\begin{aligned}
& \dot{x}=-a x y-a g x y^{2} \\
& \dot{y}=a x y-b y-b g y z \\
& \dot{z}=b y+b g y^{2},
\end{aligned}
$$

and its constants of motion are given by (6); namely,

$$
\begin{aligned}
& C_{1}(x, y, z)=x+y+z+\frac{g}{2} y^{2}+\frac{g}{2} z^{2}, \\
& I_{2}(x, y, z)=\ln x+\frac{a}{b} z .
\end{aligned}
$$

In what follows we need constants of motion defined on $\mathbb{R}^{3}$. We immediately get that the function $C_{2}$,

$$
C_{2}(x, y, z)=x \cdot e^{(a / b) z},
$$

is a constant of motion of system (10).

3.1. Hamilton-Poisson Realizations. We recall that the dynamical system generated by the $C^{1}$ vector field $\mathbf{f}=\left(f_{1}, f_{2}, f_{3}\right)$ on a $C^{\infty}$ manifold $P \subseteq \mathbb{R}^{3}$,

$$
\dot{\mathbf{x}}=\mathbf{f}(\mathbf{x}), \quad \mathbf{x}=\left(x_{1}, x_{2}, x_{3}\right),
$$

has the Hamilton-Poisson realization $(P,\{\cdot, \cdot\}, H)$, if it can be put in the form

$$
\dot{x}_{i}=\left\{x_{i}, H\right\}, \quad i \in\{1,2,3\},
$$

where $H$ is the Hamiltonian function, and $\{\cdot, \cdot\}$ is a Poisson bracket on $P$.

Considering a smooth function $C$ on $P$, a Poisson structure on $P$ is generated by the Poisson bracket

$$
\{f, g\}_{C}:=\frac{\partial(C, f, g)}{\partial\left(x_{1}, x_{2}, x_{3}\right)}=\left|\begin{array}{lll}
\partial_{x_{1}} C & \partial_{x_{2}} C & \partial_{x_{3}} C \\
\partial_{x_{1}} f & \partial_{x_{2}} f & \partial_{x_{3}} f \\
\partial_{x_{1}} g & \partial_{x_{2}} g & \partial_{x_{3}} g
\end{array}\right|
$$

for every $f, g \in C^{\infty}\left(\mathbb{R}^{3}, \mathbb{R}\right)$. In addition, see, for example, [20], using any smooth function $\nu$ on $P$, a new Poisson bracket is given by

$$
\{f, g\}_{C}^{\nu}:=v \cdot\{f, g\}_{C} .
$$

In both cases, the function $C$ is a Casimir of the Poisson structure; that is, $\{C, f\}=0$ for every $f$. In coordinates, the Poisson structure is given in matrix notation

$$
\Pi_{C}^{v}=\nu \Pi_{C}=v\left[\begin{array}{ccc}
0 & \partial_{z} C & -\partial_{y} C \\
-\partial_{z} C & 0 & \partial_{x} C \\
\partial_{y} C & -\partial_{x} C & 0
\end{array}\right] .
$$

Moreover, if there is a smooth function $H$ such that system (13) takes the form $\dot{\mathbf{x}}^{t}=\Pi_{C}^{v} \cdot \nabla H$, then (13) is a HamiltonPoisson system.

In our case, let $g \neq 0$ and let

$$
C(x, y, z)=C_{1}(x, y, z)=x+y+z+\frac{g}{2} y^{2}+\frac{g}{2} z^{2} .
$$

We obtain the rescaling function $\nu(x, y, z)=b y \cdot e^{-(a / b) z}$ and the Poisson structure generated by $C_{1}$ :

$$
\begin{aligned}
\Pi_{C_{1}} & =\left[\begin{array}{ccc}
0 & g z+1 & -g y-1 \\
-g z-1 & 0 & 1 \\
g y+1 & -1 & 0
\end{array}\right] \\
& =\left[\begin{array}{ccc}
0 & g z & -g y \\
-g z & 0 & 0 \\
g y & 0 & 0
\end{array}\right]+\left[\begin{array}{ccc}
0 & 1 & -1 \\
-1 & 0 & 1 \\
1 & -1 & 0
\end{array}\right] .
\end{aligned}
$$


Due to its linearity, the above Poisson structure is a LiePoisson structure on the dual vector space of a Lie algebra, namely, $\mathfrak{s e}(2)^{*}$. Indeed, consider the special Euclidean Lie group $\mathrm{SE}(2)$ of all orientation-preserving isometries (see, for example, [21]), given by

$$
\begin{aligned}
\operatorname{SE}(2) & =\{A \in G L(3, \mathbb{R}) \mid A \\
& \left.=\left[\begin{array}{ccc}
1 & 0 & 0 \\
u & \cos \varphi & -\sin \varphi \\
v & \sin \varphi & \cos \varphi
\end{array}\right], u, v \in \mathbb{R}, \varphi \in[0,2 \pi)\right\} .
\end{aligned}
$$

The corresponding Lie algebra of $\operatorname{SE}(2)$ is

$$
\begin{aligned}
& \mathfrak{g e}(2)=\left\{X \in g l(3, \mathbb{R}) \mid X=\left[\begin{array}{ccc}
0 & 0 & 0 \\
\beta & 0 & -\alpha \\
\gamma & \alpha & 0
\end{array}\right], \alpha, \beta, \gamma\right. \\
& \quad \in \mathbb{R}\},
\end{aligned}
$$

with the commutator bracket $[X, Y]=X Y-Y X$. where

As vector space, $\mathfrak{g e}(2)$ has the basis $B_{\mathfrak{3 e}(2)}=\left\{e_{1}, e_{2}, e_{3}\right\}$,

$$
\begin{aligned}
& e_{1}=\left[\begin{array}{ccc}
0 & 0 & 0 \\
0 & 0 & -g \\
0 & g & 0
\end{array}\right], \\
& e_{2}=\left[\begin{array}{lll}
0 & 0 & 0 \\
1 & 0 & 0 \\
0 & 0 & 0
\end{array}\right], \\
& e_{3}=\left[\begin{array}{lll}
0 & 0 & 0 \\
0 & 0 & 0 \\
1 & 0 & 0
\end{array}\right] .
\end{aligned}
$$

We obtain the following bracket relations:

$$
\begin{aligned}
& {\left[e_{1}, e_{2}\right]=g e_{3},} \\
& {\left[e_{1}, e_{3}\right]=-g e_{2},} \\
& {\left[e_{2}, e_{3}\right]=0 .}
\end{aligned}
$$

We consider the bilinear form $\Theta: \mathfrak{g e}(2) \times \mathfrak{S e}(2) \rightarrow \mathbb{R}$ given by the matrix $\left(\Theta_{i j}\right)_{1 \leq i, j \leq 3}, \Theta_{12}=-\Theta_{21}=1, \Theta_{13}=-\Theta_{31}=-1$, and $\Theta_{23}=-\Theta_{32}=1$. By straightforward computations we obtain that $\Theta$ satisfies condition

$$
\Theta(X,[Y, Z])+\Theta(Y,[Z, X])+\Theta(Z,[X, Y])=0,
$$

for every triplet $(X, Y, Z)$ of elements in $\mathfrak{g e}(2)$. Therefore $\Theta$ is a symplectic cocycle of the Lie algebra $\mathfrak{g e}(2)$. Moreover, it is not a coboundary since $\Theta\left(e_{2}, e_{3}\right)=1 \neq 0=f\left(\left[e_{2}, e_{3}\right]\right)$, for every linear map $f, f: \mathfrak{s e}(2) \rightarrow \mathbb{R}$. Following [22], the modified Lie-Poisson structure $\Pi_{C_{1}}$ is defined on the dual space $\mathfrak{g e}(2)^{*} \simeq \mathbb{R}^{3}$.

The following result gives a Hamilton-Poisson realization of the considered system.

Proposition 2. Let $v$ be the rescaling function given by

$$
\nu(x, y, z)=b y \cdot e^{-(a / b) z} .
$$

If $\Pi_{C_{1}}$ is the Poisson structure generated by the Casimir function $C_{1}$,

$$
C_{1}(x, y, z)=x+y+z+\frac{g}{2} y^{2}+\frac{g}{2} z^{2}
$$

given by

$$
\begin{aligned}
& \{x, y\}_{C_{1}}=g z+1, \\
& \{x, z\}_{C_{1}}=-g y-1, \\
& \{y, z\}_{C_{1}}=1,
\end{aligned}
$$

then system (10) has the Hamilton-Poisson realization

$$
\left(\mathbb{R}^{3},\{\cdot, \cdot\}_{C_{1}}^{v}, C_{2}\right),
$$

where $\{f, g\}_{C_{1}}^{\nu}=v \cdot\{f, g\}_{C_{1}}$ for any $f, g \in C^{\infty}\left(\mathbb{R}^{3}, \mathbb{R}\right)$, and $C_{2}$ is the Hamiltonian function given by

$$
C_{2}(x, y, z)=x \cdot e^{(a / b) z} .
$$

Proof. An easy computation shows that system (10) takes the form $\Pi_{C_{1}}^{v} \cdot \nabla C_{2}=(\dot{x}, \dot{y}, \dot{z})^{t}$; hence the conclusion follows.

Using the same notations as in Proposition 2, we similarly obtain the following results.

Proposition 3. Let $\mu$ be the rescaling function given by $\mu(x, y, z)=-v(x, y, z)$ and let $C_{2}$ be a Casimir function. If $\Pi_{C_{2}}^{\mu}$ is the Poisson structure generated by $C_{2}$ and $\mu$, given in matrix notation by

$$
\Pi_{C_{2}}^{\mu}=\left[\begin{array}{ccc}
0 & -a x y & 0 \\
a x y & 0 & -b y \\
0 & b y & 0
\end{array}\right],
$$

then system (10) has the Hamilton-Poisson realization

$$
\left(\mathbb{R}^{3},\{\cdot, \cdot\}_{C_{2}}^{\mu}, C_{1}\right),
$$

where $C_{1}$ is the Hamiltonian function.

Remark 4. The above Poisson structures are compatible and

$$
\Pi_{C_{1}}^{v} \nabla C_{2}=\Pi_{C_{2}}^{\mu} \nabla C_{1},
$$

and hence (10) is a bi-Hamiltonian system. Moreover, this pair of Hamilton-Poisson realizations gives rise to infinitely many Hamilton-Poisson realizations of system (10) (see Proposition 5). 
Proposition 5. Let $p, q, r, s \in \mathbb{R}$ such that $p s-q r=1$, and let $v(x, y, z)=b y \cdot e^{-(a / b) z}$ be the rescaling function. There exists infinitely many Hamilton-Poisson realizations of system (10) given by $\left(\mathbb{R}^{3}, \Pi_{p, q}, H_{r, s}\right)$, where the Hamiltonian $H_{r, s}$ is given by

$$
\begin{aligned}
H_{r, s} & =r C_{1}+s C_{2} \\
& =r\left(x+y+z+\frac{g}{2} y^{2}+\frac{g}{2} z^{2}\right)+s x \cdot e^{(a / b) z},
\end{aligned}
$$

the Poisson structure is defined by

$$
\Pi_{p, q}=p \Pi_{C_{1}}^{v}+q \Pi_{C_{2}}^{v},
$$

and a Casimir function of the above Poisson structure is given by

$$
\begin{aligned}
C_{p, q} & =p C_{1}+q C_{2} \\
& =p\left(x+y+z+\frac{g}{2} y^{2}+\frac{g}{2} z^{2}\right)+q x \cdot e^{(a / b) z} .
\end{aligned}
$$

Proof. It is clear that $\Pi_{p, q}=v\left(p \Pi_{C_{1}}+q \Pi_{C_{2}}\right)$. Therefore $\Pi_{p, q}$ is the Poisson structure generated by the Casimir function $C_{p, q}$ and the rescaling function $\nu$. Using the Hamilton-Poisson realizations given in Propositions 2 and 3, we immediately obtain $\Pi_{p, q} \cdot \nabla H_{r, s}=(\dot{x}, \dot{y}, \dot{z})^{t}$, which finishes the proof.

3.2. Stability of the Equilibrium Points. The equilibrium points of system (10) are given by the following families:

$$
\begin{aligned}
& \mathscr{E}_{1}=\left\{\left(M,-\frac{1}{g}, \frac{a M-b}{b g}\right): M \in \mathbb{R}\right\}, \\
& \mathscr{E}_{2}=\{(N, 0, P): N, P \in \mathbb{R}\} .
\end{aligned}
$$

We remark that the second family represents the set of all equilibrium points of Kermack-McKendrick system (2). Therefore, the existence of another family of equilibrium points produces changes in the dynamics of initial system (2). We are concerned with the study of these new equilibrium points.

Proposition 6. Let $g \in \mathbb{R} \backslash\{0\}$ and let $e_{1}^{M}=(M,-1 / g$, (aMb) $/ \mathrm{bg}) \in \mathscr{E}_{1}$ be an equilibrium point of system (10). point.

(i) If $g\left(a^{2} M+b^{2} g\right)<0$, then $e_{1}^{M}$ is an unstable equilibrium

(ii) If $g\left(a^{2} M+b^{2} g\right)>0$, then $e_{1}^{M}$ is a nonlinearly stable equilibrium point.

Proof. (i) Let $A$ be the matrix of the linear part of our system; that is,

$$
A=\left[\begin{array}{ccc}
-a y(1+g y) & -a x-2 a g x & 0 \\
a y & a x-b-b g z & -b g y \\
0 & b+2 b g y & 0
\end{array}\right] .
$$

The characteristic roots of $A\left(e_{1}^{M}\right)$ are given by

$$
\begin{aligned}
\lambda_{1} & =0, \\
\lambda_{2,3} & = \pm \sqrt{-b^{2}-\frac{a^{2} M}{g}} .
\end{aligned}
$$

Considering $M$ such that $g\left(a^{2} M+b^{2} g\right)<0$, we conclude that the equilibrium point $e_{1}^{M}$ is unstable.

(ii) Now, let $M$ be such that $g\left(a^{2} M+b^{2} g\right)>0$. We use the energy-Casimir method [23]. Let $H_{\varphi}$ be the energy-Casimir function:

$$
\begin{aligned}
H_{\varphi}(x, y, z)= & x+y+z+\frac{g}{2} y^{2}+\frac{g}{2} z^{2} \\
& +\varphi\left(x \cdot e^{(a / b) z}\right)
\end{aligned}
$$

where $\varphi: \mathbb{R} \rightarrow \mathbb{R}$ is a smooth real valued function defined on $\mathbb{R}$.

The first variation of $H_{\varphi}$ is given by

$$
\begin{aligned}
\delta H_{\varphi} & (x, y, z) \\
= & {\left[1+e^{(a / b) z} \cdot \dot{\varphi}\left(x \cdot e^{(a / b) z}\right)\right] \delta x+(1+g y) \delta y } \\
\quad+ & {\left[1+g z+\frac{a x}{b} \cdot e^{(a / b) z} \cdot \dot{\varphi}\left(x \cdot e^{(a / b) z}\right)\right] \delta z, }
\end{aligned}
$$

where $\dot{\varphi}(u):=d \varphi / d u$. We have

$$
\begin{gathered}
\delta H_{\varphi}\left(e_{1}^{M}\right)=\left[1+e^{a(a M-b) / b^{2} g} \cdot \dot{\varphi}\left(M \cdot e^{a(a M-b) / b^{2} g}\right)\right] \\
\cdot\left(\delta x+\frac{a M}{b} \delta z\right),
\end{gathered}
$$

which vanishes if and only if

$$
\dot{\varphi}\left(M \cdot e^{a(a M-b) / b^{2} g}\right)=-e^{-a(a M-b) / b^{2} g} .
$$

The second variation of $H_{\varphi}$ is given by

$$
\begin{aligned}
& \delta^{2} H_{\varphi}(x, y, z)=e^{(2 a / b) z} \cdot \ddot{\varphi}\left(x \cdot e^{(a / b) z}\right)(\delta x)^{2} \\
& +g(\delta y)^{2}+x \cdot \frac{a^{2}}{b^{2}} \cdot e^{(a / b) z}\left[g+\dot{\varphi}\left(x \cdot e^{(a / b) z}\right)+x\right. \\
& \left.\cdot e^{(a / b) z} \ddot{\varphi}\left(x \cdot e^{(a / b) z}\right)\right](\delta z)^{2}+\frac{2 a}{b} \\
& \cdot e^{(a / b) z}\left[\dot{\varphi}\left(x \cdot e^{(a / b) z}\right)+x \cdot e^{(a / b) z} \ddot{\varphi}\left(x \cdot e^{(a / b) z}\right)\right] \\
& \cdot \delta x \delta z .
\end{aligned}
$$

Taking into account relation (42), we obtain

$$
\begin{aligned}
& \delta^{2} H_{\varphi}\left(e_{1}^{M}\right)=e^{2 a(a M-b) / b^{2} g} \cdot \ddot{\varphi}\left(M \cdot e^{a(\mathrm{a} M-b) / b^{2} g}\right) \\
& \cdot(\delta x)^{2}+g(\delta y)^{2}+\left[g-M \cdot \frac{a^{2}}{b^{2}}+M^{2} \cdot \frac{a^{2}}{b^{2}}\right. \\
& \left.\cdot e^{2 a(a M-b) / b^{2} g} \cdot \ddot{\varphi}\left(M \cdot e^{a(a M-b) / b^{2} g}\right)\right](\delta z)^{2} \\
& +\frac{2 a}{b}\left[M \cdot e^{2 a(a M-b) / b^{2} g} \cdot \ddot{\varphi}\left(M \cdot e^{a(a M-b) / b^{2} g}\right)-1\right] \\
& \cdot \delta x \delta z .
\end{aligned}
$$


If $g>0$, then we choose a function $\varphi$ such that relation (42) holds and

$$
\begin{aligned}
\ddot{\varphi}\left(M \cdot e^{a(a M-b) / b^{2} g}\right) & >\frac{a^{2}}{b^{2} g+a^{2} M} \cdot e^{-2 a(a M-b) / b^{2} g} \\
& >0 .
\end{aligned}
$$

For example, let

$$
\begin{aligned}
\varphi(u)= & \frac{1}{2}\left(g+\frac{a^{2}}{b^{2} g+a^{2} M} \cdot e^{-2 a(a M-b) / b^{2} g}\right) \\
& \cdot\left(u-M \cdot e^{a(a M-b) / b^{2} g}\right)^{2}-e^{-a(a M-b) / b^{2} g} u .
\end{aligned}
$$

We get that $\delta^{2} H_{\varphi}\left(e_{1}^{M}\right)$ is positive definite. Therefore the equilibrium point $e_{1}^{M}$ is nonlinearly stable.

If $g<0$, then the same function $\varphi$ has the property

$$
\begin{aligned}
\ddot{\varphi}\left(M \cdot e^{a(a M-b) / b^{2} g}\right) & <\frac{a^{2}}{b^{2} g+a^{2} M} \cdot e^{-2 a(a M-b) / b^{2} g} \\
& <0 .
\end{aligned}
$$

We obtain that $\delta^{2} H_{\varphi}\left(e_{1}^{M}\right)$ is negative definite, and, in consequence, the equilibrium point $e_{1}^{M}$ is nonlinearly stable.

Remark 7. Let $e_{2}^{N, P}=(N, 0, P) \in \mathscr{E}_{2}$. The eigenvalues of the characteristic polynomial associated with the linearization of system (10) at $e_{2}^{N, P}$ are given by $\lambda_{1,2}=0, \lambda_{3}=a N-b-b g P$. Therefore the equilibrium point $e_{2}^{N, P}$ is unstable in the case $a N-b-b g P>0$.

3.3. Energy-Casimir Mapping. We consider the HamiltonPoisson realization of system (10) given in Proposition 3. The corresponding energy-Casimir mapping $\mathscr{E} \mathscr{C}: \mathbb{R}^{3} \rightarrow \mathbb{R}^{2}$ is given by

$$
\begin{aligned}
\mathscr{E} \mathscr{C}(x, y, z) & =(H(x, y, z), C(x, y, z)) \\
& =\left(x+y+z+\frac{g}{2} y^{2}+\frac{g}{2} z^{2}, x \cdot e^{(a / b) z}\right) .
\end{aligned}
$$

The set

$$
\begin{aligned}
\operatorname{Im}(\mathscr{E} \mathscr{C}) & =\left\{(h, c) \in \mathbb{R}^{2} \mid(\exists)(x, y, z)\right. \\
& \left.\in \mathbb{R}^{3}: H(x, y, z)=h, C(x, y, z)=c\right\}
\end{aligned}
$$

is called the image of the energy-Casimir mapping.

We denote by $\Gamma_{M}$ the set of images of the equilibrium points $(M,-1 / g,(a M-b) / b g)$ through the energy-Casimir mapping; namely,

$$
\begin{aligned}
\Gamma_{M} & :=\left\{(h, c) \in \mathbb{R}^{2}: h=M-\frac{1}{g}+\frac{a^{2}}{2 b^{2} g} M^{2}, c=M\right. \\
\cdot & \left.e^{\left(a^{2} / b^{2} g\right) M-a / b g}, M \in \mathbb{R}\right\} .
\end{aligned}
$$

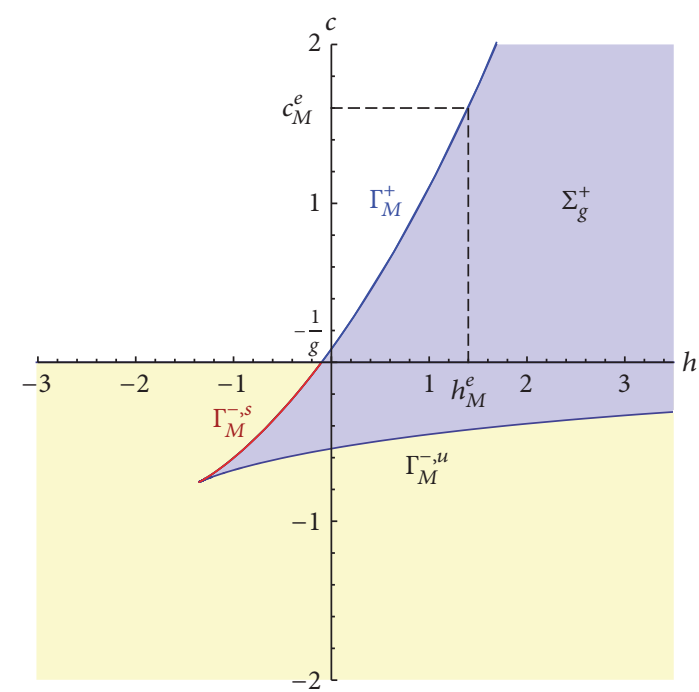

FIGURE 1: The image of the energy-Casimir mapping ( $g=10, a=$ $2, b=1)$.

We also consider the subsets

$$
\begin{aligned}
& \Gamma_{M}^{+}:=\left\{(h, c) \in \Gamma_{M}: M>0\right\}, \\
& \Gamma_{M}^{-}:=\left\{(h, c) \in \Gamma_{M}: M<0\right\} .
\end{aligned}
$$

For $g>0$, we deduce that $\Gamma_{M}^{+}$is the graph of a function $c=$ $\varphi(h), h>-1 / g$. Also, for $g<0$, we have that $\Gamma_{M}^{-}$is the graph of a function $c=\psi(h), h<-1 / g$. We define the sets

$$
\Sigma_{g}^{+}:=\left\{(h, c) \in \mathbb{R}^{2}: h>-\frac{1}{g}, 0<c<\varphi(h)\right\}
$$

for $g>0$,

$$
\Sigma_{g}^{-}:=\left\{(h, c) \in \mathbb{R}^{2}: h<-\frac{1}{g}, \psi(h)<c<0\right\}
$$

for $g<0$.

The set $\operatorname{Im}(\mathscr{E} \mathscr{C})$ is described in the next result.

Proposition 8. (i) Let $g>0$. The image of the energy-Casimir mapping $\mathscr{E} \mathscr{C}$ (48) is given by

$$
\begin{aligned}
\operatorname{Im}(\mathscr{E} \mathscr{C})= & \{(h, c): c<0\} \\
& \cup\left\{(h, c): h \geq-\frac{1}{g}, c=0\right\} \cup \Sigma_{g}^{+} \cup \Gamma_{M}^{+},
\end{aligned}
$$

where $\Gamma_{M}^{+}$and $\Sigma_{g}^{+}$are given by (51) and (53), respectively (Figure 1).

(ii) Let $g<0$. The image of the energy-Casimir mapping $\mathscr{E} \mathscr{C}$ (48) is given by

$$
\begin{aligned}
\operatorname{Im}(\mathscr{E} \mathscr{C})= & \{(h, c): c>0\} \\
& \cup\left\{(h, c): h \leq-\frac{1}{g}, c=0\right\} \cup \Sigma_{g}^{-} \cup \Gamma_{M}^{-},
\end{aligned}
$$




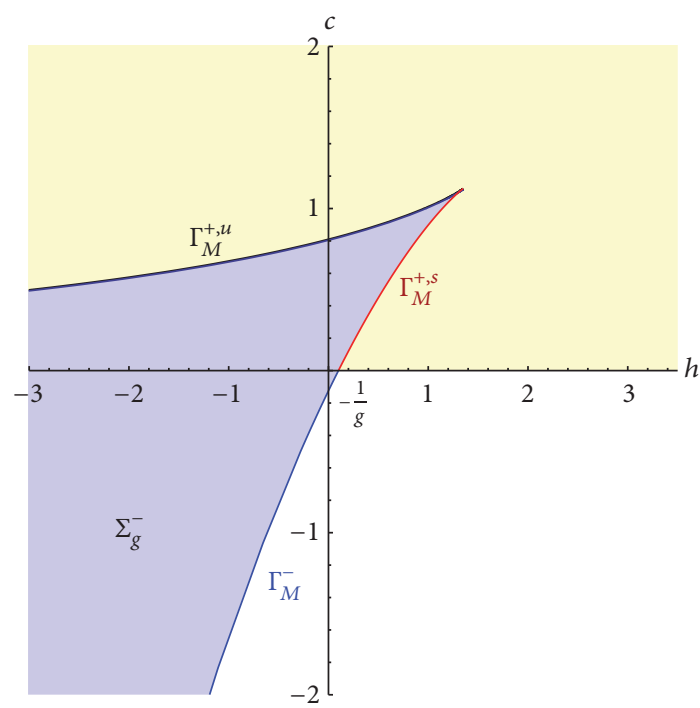

Figure 2: The image of the energy-Casimir mapping $(g=-10, a=$ $2, b=1)$.

where $\Gamma_{M}^{-}$and $\Sigma_{g}^{-}$are given by (52) and (54), respectively (Figure 2).

Proof. (i) Let $h \in \mathbb{R}, c \in(-\infty, 0)$, arbitrary. Consider $y=$ $-1 / g$. The conditions $H(x, y, z)=h, C(x, y, z)=c$ from (49) become

$$
\begin{aligned}
& x=h+\frac{1}{g}-\frac{g}{2}\left(z+\frac{1}{g}\right)^{2}:=f_{1}(z) \\
& x=c \cdot e^{-(a / b) z}:=f_{2}(z) .
\end{aligned}
$$

We deduce that the image of the function $f=f_{1}-f_{2}$ is $\mathbb{R}$; hence the above system has solution for every $h$ and $c$. Therefore $\{(h, c): c<0\} \subset \operatorname{Im}(\mathscr{E} \mathscr{C})$.

The condition $c=0$ implies $x=0$; hence the equation $H(x, y, z)=h$ has solution if and only if $h \geq-1 / g$; that is, $\{(h, c): h \geq-1 / g, c=0\} \subset \operatorname{Im}(\mathscr{E} \mathscr{C})$.

It is clear that $\Gamma_{M}^{+} \subset \operatorname{Im}(\mathscr{E} \mathscr{C})$. It only remains to prove $\Sigma_{g}^{+} \subset \operatorname{Im}(\mathscr{E} \mathscr{C})$ and other pairs $(h, c)$ do not belong to $\operatorname{Im}(\mathscr{E} \mathscr{C})$.

Consider the functions

$$
\begin{aligned}
& u(z)=M+\frac{a^{2} M^{2}}{2 b^{2} g}-\frac{g}{2}\left(z+\frac{1}{g}\right)^{2}, \\
& v(z)=M \cdot e^{(a / b)(a M / b g-1 / g-z)}, \\
& f(z)=u(z)-v(z) .
\end{aligned}
$$

We deduce that there is $z_{0} \in \mathbb{R}$ such that $f\left(z_{0}\right)=0$ and $f(z)<$ 0 for all $z \in \mathbb{R} \backslash\left\{z_{0}\right\}$. Moreover, $\operatorname{Im}(f)=(-\infty, 0]$.

Now, we fix an arbitrary pair $\left(h_{M}^{e}, c_{M}^{e}\right) \in \Gamma_{M}^{+}$, where $h_{M}^{e}=$ $M-1 / g+\left(a^{2} / 2 b^{2} g\right) M^{2}, c_{M}^{e}=M \cdot e^{\left(a^{2} / b^{2} g\right) M-a / b g}, M>0$. We show that $\left(h, c_{M}^{e}\right) \notin \operatorname{Im}(\mathscr{E} \mathscr{C})$ for every $h<h_{M}^{e}$ and $\left(h, c_{M}^{e}\right) \in$ $\operatorname{Im}(\mathscr{E} \mathscr{C})$ for every $h>h_{M}^{e}$.
With the above notations, for a pair $\left(h, c_{M}^{e}\right)$ the system given by (49) becomes

$$
\begin{aligned}
x & =h+\frac{1}{g}-\frac{g}{2}\left(y+\frac{1}{g}\right)^{2}-\frac{g}{2}\left(z+\frac{1}{g}\right)^{2} \\
& =u(z)+h-h_{M}^{e}-\frac{g}{2}\left(y+\frac{1}{g}\right)^{2} \\
x & =c_{M}^{e} \cdot e^{-(a / b) z}=v(z),
\end{aligned}
$$

and hence

$$
f(z)+h-h_{M}^{e}=\frac{g}{2}\left(y+\frac{1}{g}\right)^{2} .
$$

Because $\operatorname{Im}(f)=(-\infty, 0]$ we obtain that there is $z$ such that $f(z)+h-h_{M}^{e} \geq 0$ for any $h>h_{M}^{e}$. Consequently, (60) has solution; that is, $\left(h, c_{M}^{e}\right) \in \operatorname{Im}(\mathscr{E} \mathscr{C})$ for every $h>h_{M}^{e}$. Therefore $\Sigma_{g}^{+} \subset \operatorname{Im}(\mathscr{E} \mathscr{C})$.

On the other hand, we get that (60) does not have solutions for $h<h_{M}^{e}$. Therefore $\left(h, c_{e}\right) \notin \operatorname{Im}(\mathscr{E} \mathscr{C})$ for every $h<h_{M}^{e}$, which finishes the proof of (i).

(ii) The conclusion follows using the same arguments as in the first case.

Remark 9. The image of the energy-Casimir mapping is a nonconvex subset of $\mathbb{R}^{2}$. Moreover, it is not a closed set, and, clearly, it is not the convex hull of the set of the images of the stable equilibrium points of the system through the map $\mathscr{E} \mathscr{C}$.

Taking into account the results that have been reported in the papers [5-9], we notice that our example shows there is no a general result regarding the properties of the image of the energy-Casimir mapping. Furthermore, the answer to the question "can $\operatorname{Im}(\mathscr{E} \mathscr{C})$ be a nonconvex set?" is affirmative.

Because one of the constants of motion is not a polynomial function, it remains an open problem to establish that the results observed in the above-mentioned papers are true in the cases when the constants of motion are polynomials.

Remark 10. Another property that has been reported is the following. As a closed set, the set $\operatorname{Im}(\mathscr{C} \mathscr{C})$ has the boundary given by images of some stable equilibrium points of the system through the energy-Casimir mapping. In our case, this property is partially true, in the sense that only a part of the boundary of $\operatorname{Im}(\mathscr{E} \mathscr{C})$, namely, the set $\Gamma_{M}^{+}(51)$, is formed by the images of stable equilibrium points through $\mathscr{E} \mathscr{C}(g>0$, see Figure 1). If $g<0$, a similar result is obtained for the set $\Gamma_{M}^{-}(52)$ (see Figure 2).

Remark 11. It is easy to see that the image through the energyCasimir mapping of a family of equilibrium points that has the form $\mathscr{E}(M)=(x(M), y(M), z(M)), M \in \mathbb{R}$, is a curve included in $\operatorname{Im}(\mathscr{E} \mathscr{C})$. In our case, for $g>0$, we have $\mathscr{E} \mathscr{C}\left(\mathscr{E}_{1}\right)=\Gamma_{M}^{+} \cup \Gamma_{M}^{-, s} \cup \Gamma_{M}^{-, u}$ (Figure 1), where the superscripts $s$ and $u$ mean stable and unstable, respectively. On the other hand, the second family of equilibrium points depends of two parameters. It is natural to ask about the image of this family through the energy-Casimir mapping. 


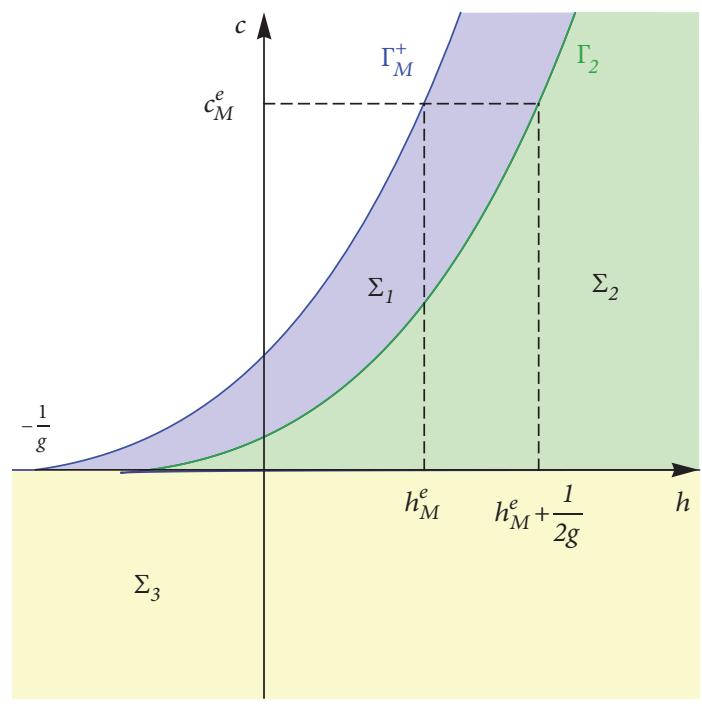

Figure 3: The images of the equilibrium points $(N, 0, P)$ through the energy-Casimir mapping: $\Sigma_{2} \cup \Sigma_{3}(g=1, a=2, b=1)$.

In the next result we give the set of all images of the equilibrium points that belong to $\mathscr{E}_{2}$ through the energyCasimir mapping.

Proposition 12. (i) Let $g>0$.

(a) For all $(h, c) \in \operatorname{Im}(\mathscr{E} \mathscr{C}), c<0$, there is an equilibrium point $(N, 0, P)$ such that $\mathscr{E} \mathscr{C}(N, 0, P)=(h, c)$.

(b) For every $c \geq 0$, let $h_{M}^{e}=M-1 / g+\left(a^{2} / 2 b^{2} g\right) M^{2}$ such that $c=c_{M}^{e}=M \cdot e^{\left(a^{2} / b^{2} g\right) M-a / b g}, M \geq 0$ (see Figure 3$)$. Then for every $h \geq h_{M}^{e}+1 / 2 g$ there is an equilibrium point $(N, 0, P)$ such that $\mathscr{E} \mathscr{C}(N, 0, P)=\left(h, c_{M}^{e}\right)$.

(ii) Let $g<0$.

(a) For all $(h, c) \in \operatorname{Im}(\mathscr{E} \mathscr{C}), c>0$, there is an equilibrium point $(N, 0, P)$ such that $\mathscr{E} \mathscr{C}(N, 0, P)=(h, c)$.

(b) For every $c \leq 0$, let $h_{M}^{e}=M-1 / g+\left(a^{2} / 2 b^{2} g\right) M^{2}$ such that $c=c_{M}^{e}=M \cdot e^{\left(a^{2} / b^{2} g\right) M-a / b g}, M \leq 0$. Then for every $h \leq h_{M}^{e}+1 / 2 g$ there is an equilibrium point $(N, 0, P)$ such that $\mathscr{E} \mathscr{C}(N, 0, P)=\left(h, c_{M}^{e}\right)$.

Proof. (i) We have

$$
\mathscr{E} \mathscr{C}(N, 0, P)=\left(N+P+\frac{g}{2} P^{2}, N \cdot e^{(a / b) P}\right)=(h, c),
$$

and hence

$$
\begin{aligned}
N & =c \cdot e^{-(a / b) P}, \\
c \cdot e^{-(a / b) P}+P+\frac{g}{2} P^{2} & =h .
\end{aligned}
$$

We denote

$$
f(P)=c \cdot e^{-(a / b) P}+P+\frac{g}{2} P^{2}-h .
$$

(a) If $c<0$, then the image of the function $f$ is $\mathbb{R}$. Therefore there is $P \in \mathbb{R}$ such that $f(P)=0$. Consequently, there is an equilibrium point $(N, 0, P)$ such that $\mathscr{E} \mathscr{C}(N, 0, P)=(h, c)$. (b) For each $M \geq 0$, let $\left(h_{M}^{e}, c_{M}^{e}\right) \in \Gamma_{M}^{+}$; that is, $h_{M}^{e}=$ $M-1 / g+\left(a^{2} / 2 b^{2} g\right) M^{2}, c_{M}^{e}=M \cdot e^{\left(a^{2} / b^{2} g\right) M-a / b g}$. If $c=c_{M}^{e}$, then the function $f(63)$ becomes

$$
f(P)=M \cdot e^{(a / b)(a M / b g-1 / g-P)}+P+\frac{g}{2} P^{2}-h .
$$

We obtain $\operatorname{Im} f=\left[h_{M}^{e}+1 / 2 g-h, \infty\right)$, where $h_{M}^{e}+1 / 2 g-$ $h=f(a M / b g-1 / g)$. Therefore the equation $f(P)=0$ has solutions if and only if $h \geq h_{M}^{e}+1 / 2 g$, and conclusion follows.

(ii) It is analogous.

Remark 13. Because $\mathscr{E} \mathscr{C}\left(\mathscr{E}_{2}\right) \subset \operatorname{Im}(\mathscr{E} \mathscr{C})$, from the above Proposition we deduce that if $g>0$, then $\mathscr{E} \mathscr{C}\left(\mathscr{E}_{2}\right)=\Sigma_{2} U$ $\Sigma_{3}$, where $\Sigma_{2}$ contains all the points $(h, c)$ situated within $\mathscr{E} \mathscr{C}(0,0, P)$, that is, $c=0, h \geq-1 / 2 g$, and $\mathscr{E} \mathscr{C}(M, 0, a M / b g-$ $1 / g), M \geq 0$, that is, $\Gamma_{2}$ (see Figure 3 ). A similar result is obtained in the case $g<0$.

\section{Conclusions}

In [4], Tudoran et al. have considered the energy-Casimir mapping associated with a Hamilton-Poisson system and have proposed an open problem regarding the connections between dynamical properties of a Hamilton-Poisson system and the corresponding energy-Casimir mapping. The observed properties remain true for some particular systems [5-9]. It was natural to ask if there are other cases [12]. In our paper, we have considered such a case, obtained by using integrable deformations of the Kermack-McKendrick model. We have given Hamilton-Poisson realizations of the considered system. We have also studied the stability of the new family of equilibrium points that has developed in the considered dynamics. Furthermore, we have pointed out some properties of the energy-Casimir mapping associated with the considered system.

In our case, the image of the energy-Casimir mapping has other properties than those reported for other systems, which leaves room for further studies such as the existence of the periodic orbits of the considered system around some nonlinearly stable equilibrium points that belong to the first family, as well as homoclinic and heteroclinic orbits.

\section{Data Availability}

No data were used to support this study.

\section{Conflicts of Interest}

The authors declare that there are no conflicts of interest regarding the publication of this paper.

\section{Acknowledgments}

This work was supported by Research Grant PCD-TC-2017. 


\section{References}

[1] A. Galajinsky, "Remark on integrable deformations of the Euler top," Journal of Mathematical Analysis and Applications, vol. 416, no. 2, pp. 995-997, 2014.

[2] C. Lăzureanu, "On the Hamilton-Poisson realizations of the integrable deformations of the Maxwell-Bloch equations," Comptes Rendus Mathematique, vol. 355, no. 5, pp. 596-600, 2017.

[3] C. Lăzureanu, "Hamilton-Poisson realizations of the integrable deformations of the Rikitake system," Advances in Mathematical Physics, Article ID 4596951, 9 pages, 2017.

[4] R. M. Tudoran, A. Aron, and S. Nicoara, "On a Hamiltonian version of the Rikitake system," SIAM Journal on Applied Dynamical Systems, vol. 8, no. 1, pp. 454-479, 2009.

[5] C. Lăzureanu and T. Bînzar, "A Rikitake type system with quadratic control," International Journal of Bifurcation and Chaos, vol. 22, no. 11, Article ID 1250274, 14 pages, 2012.

[6] C. Lăzureanu and T. Bînzar, "Some geometrical properties of the Maxwell-Bloch equations with a linear control," in Proceedings of the in Proceedings of the 13th International Conference of Mathematics and Its Applications, pp. 151-158, Timisoara, Romania, November 2012.

[7] T. Bînzar and C. Lăzureanu, "A Rikitake type system with one control," Discrete and Continuous Dynamical Systems - Series B, vol. 18, no. 7, pp. 1755-1776, 2013.

[8] C. Lăzureanu and T. Bînzar, "Symmetries and properties of the energy-Casimir mapping in the ball-plate problem," Advances in Mathematical Physics, Article ID 5164602, 13 pages, 2017.

[9] C. Lăzureanu, “The real-valued Maxwell-Bloch equations with controls: from a Hamilton-Poisson system to a chaotic one," International Journal of Bifurcation and Chaos, vol. 27, no. 9, Article ID 1750143, 17 pages, 2017.

[10] R. M. Tudoran and A. Gîrban, "On a Hamiltonian version of a three-dimensional Lotka-Volterra system," Nonlinear Analysis: Real World Applications, vol. 13, no. 5, pp. 2304-2312, 2012.

[11] T. Bînzar and C. Lăzureanu, "On some dynamical and geometrical properties of the Maxwell-Bloch equations with a quadratic control," Journal of Geometry and Physics, vol. 70, pp. 1-8, 2013.

[12] C. Lăzureanu, "On a Hamilton-Poisson approach of the Maxwell-Bloch equations with a control," Mathematical Physics, Analysis and Geometry. An International Journal Devoted to the Theory and Applications of Analysis and Geometry to Physics, vol. 20, no. 3, Art. 20, 22 pages, 2017.

[13] W. O. Kermack and A. G. McKendrick, "A Contribution to the Mathematical Theory of Epidemics, Proceedings of the Royal Society A: Mathematical," vol. 115, pp. 700-721, 1927.

[14] R. M. Anderson and R. M. May, "Population biology of infectious diseases: part I," Nature, vol. 280, no. 5721, pp. 361367, 1979.

[15] N. Bacaër, "The model of Kermack and McKendrick for the plague epidemic in Bombay and the type reproduction number with seasonality," Journal of Mathematical Biology, vol. 64, no. 3, pp. 403-422, 2012.

[16] Y. Chen, S. Zou, and J. Yang, "Global analysis of an SIR epidemic model with infection age and saturated incidence," Nonlinear Analysis: Real World Applications, vol. 30, pp. 16-31, 2016.

[17] Z. Hu, W. Ma, and S. Ruan, "Analysis of SIR epidemic models with nonlinear incidence rate and treatment," Mathematical Biosciences, vol. 238, no. 1, pp. 12-20, 2012.
[18] H. S. Rodrigues, "Application of SIR epidemiological model: new trends," Journal of Applied Mathematics and Informatics, vol. 10, pp. 92-97, 2016.

[19] J. D. Murray, Mathematical Biology I. An Introduction, vol. 1, Springer, New York, NY, USA, 2002.

[20] R. M. Tudoran, "A normal form of completely integrable systems," Journal of Geometry and Physics, vol. 62, no. 5, pp. 1167-1174, 2012.

[21] R. M. Adams, R. Biggs, and C. C. Remsing, "Single-input control systems on the Euclidean group SE(2)," European Journal of Pure and Applied Mathematics, vol. 5, no. 1, pp. 1-15, 2012.

[22] P. Libermann and C.-M. Marle, Symplectic geometry and analytical mechanics, vol. 35 of Mathematics and its Applications, D. Reidel Publishing Co., Dordrecht, 1987.

[23] D. D. Holm, J. E. Marsden, T. Ratiu, and A. Weinstein, "Nonlinear stability of fluid and plasma equilibria," Physics Reports, vol. 123, no. 1-2, 116 pages, 1985. 


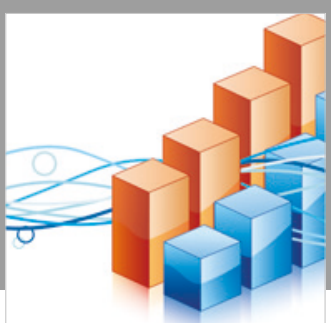

Advances in

Operations Research

\section{-n-m}
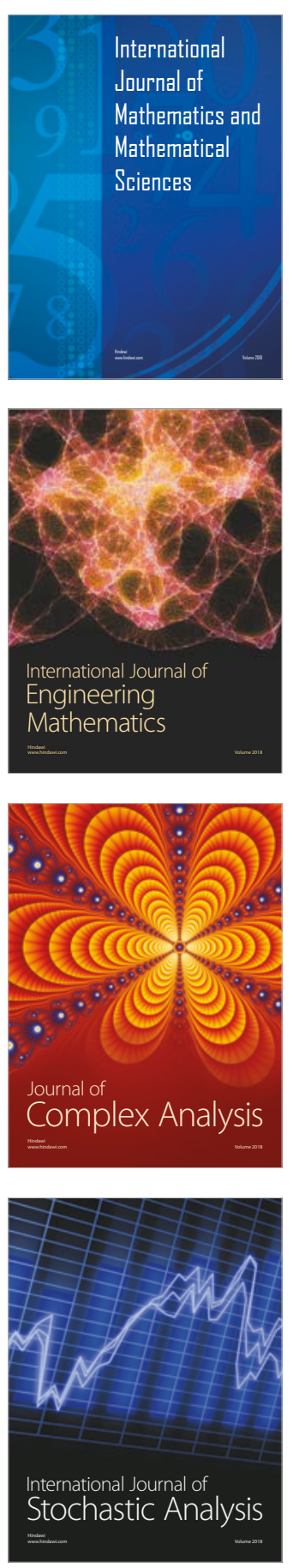
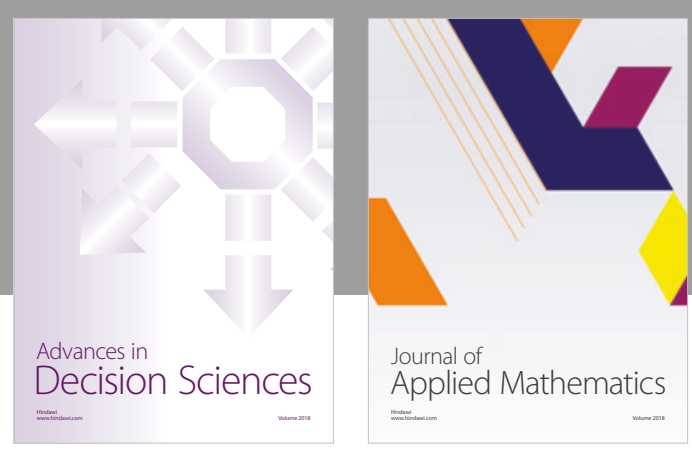

Journal of

Applied Mathematics
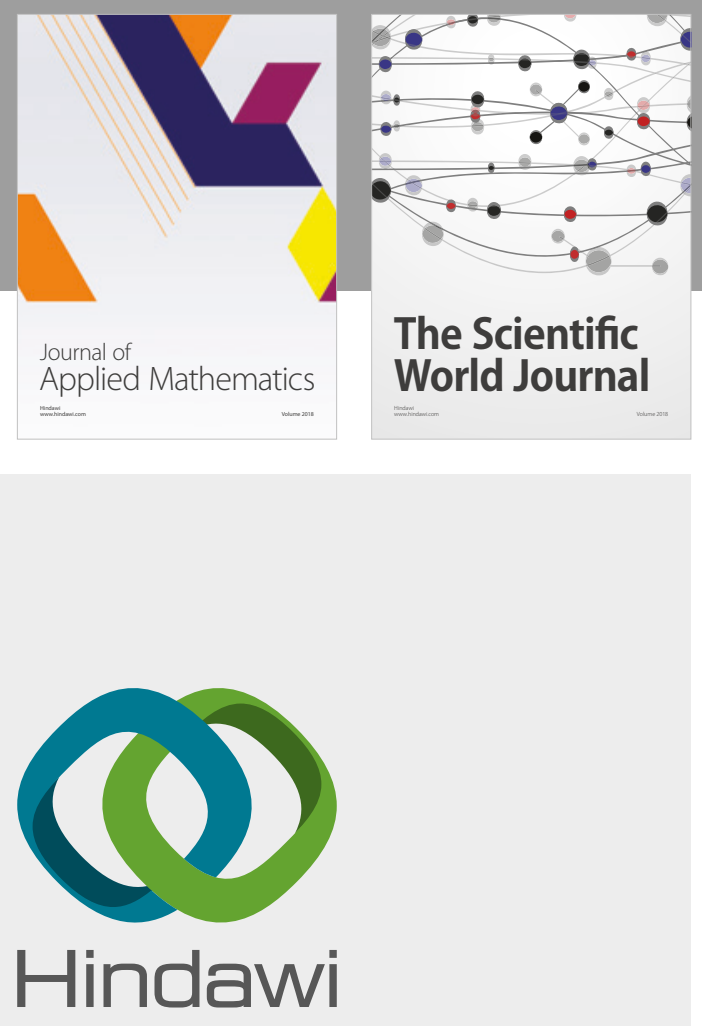

Submit your manuscripts at

www.hindawi.com

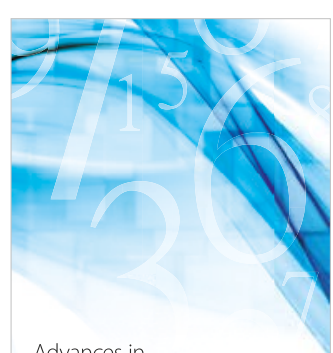

Advances in
Numerical Analysis
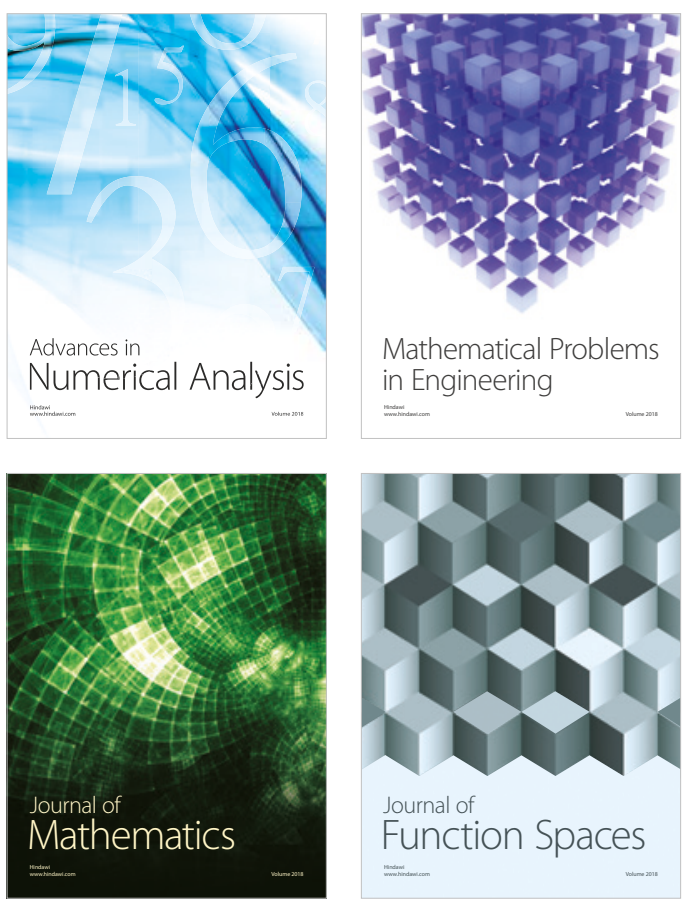

Mathematical Problems in Engineering

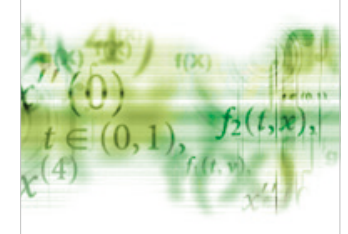

International Journal of

Differential Equations

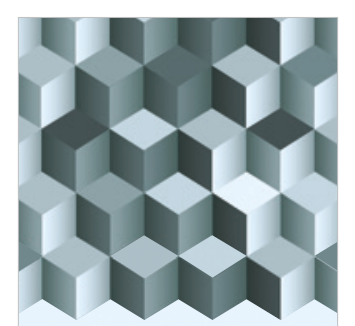

Journal of

Function Spaces

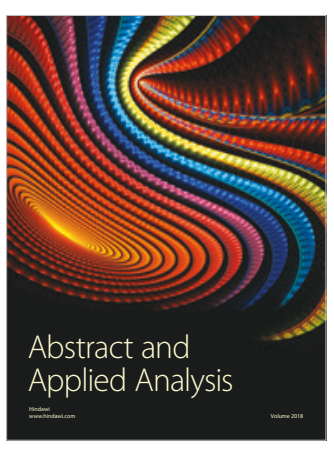

The Scientific

World Journal

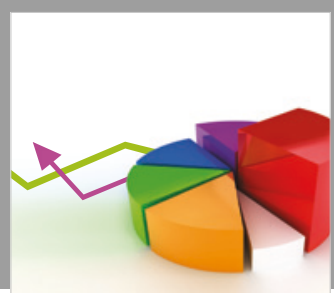

Journal of

Probability and Statistics
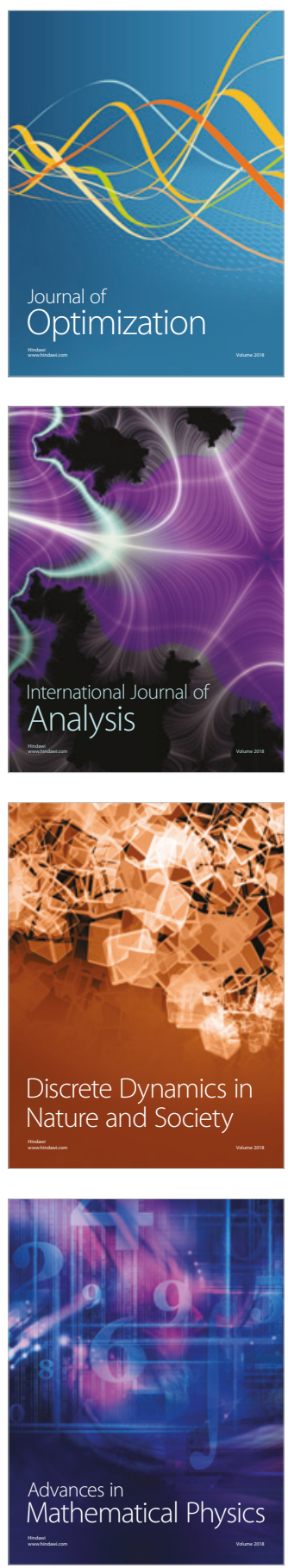